\title{
Jedes Gramm zählt
}

\begin{abstract}
Mit einem neuen Verfahren lassen sich Hohlkugeln aus metallischen und keramischen Werkstoffen ab einem Durchmesser von einem Millimeter herstellen. Im Vergleich zu einer Vollkugel werden damit Gewichtseinsparungen von bis zu $90 \%$ erzielt.
\end{abstract}

Vom Fahrzeug- und Maschinenbau über die Automatisierungs-, Mess-, Medizin- und Ventiltechnik bis zur Luft- und Raumfahrt, es gibt kaum eine Branche, in der Kugeln keine Rolle spielen. Geht es dabei um metallische Präzisionskugeln, standen üblicherweise nur massive Varianten zur Verfügung. Lediglich für besondere Anwendungen lohnte es sich, polierte Hohlkugeln aus tiefgezogenen Blechen zu fertigen. Mit diesem aufwendigen Verfahren lassen sich jedoch nur metallische Hohlkugeln ab einem Durchmesser von $10 \mathrm{~mm}$ herstellen. Die Fertigung kleinerer Formate ließ sich bisher nicht wirtschaftlich realisieren. Auch keramische Hohlkugeln sind im Markt bisher kaum verfügbar. Als Folge davon sind die Kugeln häufig überdimensioniert und deutlich schwerer als erforderlich. Das wirkt Bestrebungen zur Einsparung von Ressourcen und Energie entgegen.

\section{Anwendungsspezifisch ausgelegte Hohlkugeln}

Um dieses Problem zu lösen, hat Hollomet in Zusammenarbeit mit Ballcenter ein neues Verfahren zur reproduzierbaren Herstellung von Hohlkugeln entwickelt. Die Technologie ermöglicht, Kugeln mit definierten Wandstärken von wenigen Zehntel Millimetern, präzisen Durchmessern, Rundheiten und geringen Toleranzen ab $1 \mathrm{~mm}$ zu fertigen. Mit dem Prozess lassen sich viele in Pulverform verfügbare und sinterbare Metalle wie hochfeste Stähle und Edelstähle sowie keramische Werkstoffe wie Siliziumnitrid $\left(\mathrm{Si}_{3} \mathrm{~N}_{4}\right)$, Siliziumcarbid $(\mathrm{SiC})$, Aluminiumoxid $\left(\mathrm{Al}_{2} \mathrm{O}_{3}\right)$, Zirconiumoxid $\left(\mathrm{ZrO}_{2}\right)$ und sogar Piezokeramiken verarbeiten. Der finale Schliff erfolgt durch Ballcenter. Im Vergleich zu einer Vollkugel werden damit Gewichtseinsparungen von bis zu $90 \%$ erzielt. Gleichzeitig ermöglicht die flexible Materialauswahl, die physikalischen, chemischen und thermischen Eigenschaften der Kugeln auf die Anwendung abzustimmen. So können unter anderem Magnetismus, Temperaturbeständigkeit sowie Resistenz gegenüber Chemikalien beeinflusst werden.

\section{Hohlkugeln sparen Energie}

Durch die Anpassung an mechanische und chemische Anforderungen ergeben sich vielfältige Einsatzmöglichkeiten. Dazu zählen Messgeräte, bei denen das hohe Gewicht der bisher verwendeten Vollmaterialkugeln störende Schwingungen verursachen kann. Bei hochdrehenden Spindeln, die unter anderem in der Textilindustrie eingesetzt werden, ermöglichen die Hohlkugeln durch die Verringerung der zu bewegenden Masse Energieeinsparungen. Einen geringeren Energiever-

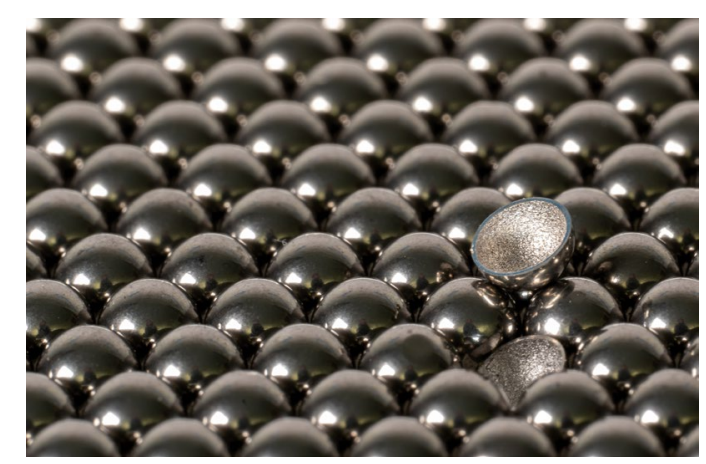

Die geschliffene Hohlkugel aus Duplexstahl (1.4462) hat eine Wandstärke von $200 \mu \mathrm{m}$ und einen Durchmesser von 3,01 mm.

(๑ hollomet) brauch bei gleichzeitig verbesserter Zustellpräzision versprechen die Hohlkugeln in der Automatisierungs- und Antriebstechnik, zum Beispiel bei Robotern und Linearsystemen sowie der Werkzeugzustellung in CNCMaschinen. Positiv wirkt sich die verringerte Kugelmasse auch auf die Reaktionszeiten von Hochgeschwindigkeitsventilen aus, die beispielsweise in der Hydraulik, Pneumatik, im Motorenbau, bei Einspritzventilen sowie in der Dosiertechnik verwendet werden. Die Messung des Füllstands oder Durchflusses von aggressiven Medien ist ebenfalls ein Einsatz, bei dem metallische und keramische Hohlkugeln Vorteile gegenüber den üblichen kunststoffbasierten Lösungen bieten können. Ein spezieller Anwendungsbereich keramischer Hohlkugeln ergibt bei der Herstellung technischer Geräte, die wie Magnetresonanztomographen empfindlich auf magnetische Werkstoffe reagieren können.

Im breiten Segment der Lagertechnik werden sehr unterschiedliche Anforderungen an die üblicherweise eingesetzten Vollmaterialkugeln gestellt, so dass sie nicht einfach durch Hohlkugeln ersetzt werden können. Allerdings lohnt es sich auch in diesem Bereich, über Leichtbaulösungen mit der gewichtsreduzierten Alternative aus Metall oder Keramik nachzudenken. Denn im Wachstumsmarkt Elektromobilität oder auch bei Drohnen, die zukünftig immer mehr Funktionen übernehmen sollen, zählt jedes Gramm.

Die Entwicklung der Kugelspezifikation erfolgt entsprechend den Anforderungen des Einsatzgebietes. Die Produktion sowie die Koordination der Abläufe von der Rohlingsbearbeitung bis zur Lagerhaltung und Disposition ist in Klein- und Großserien möglich.

Kontakt: hollomet GmbH, 01277 Dresden www.hollomet.com 